\title{
The Technology of Manufacturing for Complementary Transistors on Gallium Nitride
}

\author{
A.Yu. Pavlov, S.A. Gamkrelidze, K.N. Tomosh, Yu.V. Fedorov, V.Yu. Pavlov, \\ R.R. Galiev, P.P. Maltsev \\ Institute for Ultra High Frequency Semiconductor Electronic of RAS, Moscow, p.alex.ur@yandex.ru
}

\begin{abstract}
The need to fabricate normally-off transistors that provide operation in the enhancement mode is confirmed by publications last 3-4 years. The use of normally-off transistors in power electronics provides for a more simple and safe operation of microcircuits. Manufacture of a power and analog part on one chip will expand the electronic component base. For manufacturing of a normally-off field-effect transistor based on the AlGaN/GaN heterostructures, it is proposed to form a gate recess by a low-energy plasma-chemical etching of the AlGaN barrier layer in a high-density plasma with an inductively coupled plasma source. Etching is due to cyclic oxidation of the barrier layer with subsequent chemical removal of the oxidized layer in the chlorine-containing environment. The proposed method allows to controllably etching of the AlGaN layers with a rate of $1.5 \mathrm{~nm} /$ cycle without defect formation. Inductively coupled plasma makes it possible to obtain a plasma with high density and low energies of charged particles interacting with the sample surface. This avoids the occurrence of radiation defects in the near-surface layers of the heterostructure. The high concentration of charged particles in the reactor during the plasma chemical processes ensures high homogeneity and quality of etching. Reducing the thickness of the barrier layer of the heterostructure due to dry etching and the gate metallization ensures the operation of the transistor in the enhancement mode. The use of dry low-energy plasmachemical etching in the technological cycle of manufacturing microcircuits allows one to fabricate transistors operating in the regime of enhancement and depletion.
\end{abstract}

Keywords - normally-off transistor, gallium nitride, heterostructure, enhancement mode, depletion mode, barrier layer, plasma-chemical etching, a system on a crystal, power, microwave-high-power semiconductor electronics.

\section{INTRODUCTION}

Gallium nitride $(\mathrm{GaN})$ has proved oneself in power electronics and in microwave semiconductor electronics, due to the large band gap and high density of the main charge carriers in the heterostructure channel. HEMT $\mathrm{AlGaN} / \mathrm{GaN}$ have great potential for modern chips used to implement modern technologies and technical solutions in communication systems, radar, information transmission, expanding the capabilities of powerful high-frequency devices. The AlGaN/GaN heterostructures provide HEMT AlGaN/GaN operation in the D-mode, i.e., a standard normally-on) field-effect transistor is formed on such structures. In the formation of high-power amplifiers on normally-off nitride transistors operating in the enhancement-mode (E-mode HEMT) provides a simpler and safer operation of the chips [1]. Normally-off and normally-on transistors can also be used in logic circuits [1]. Realization of d-mode PHEMT and e-mode PHEMT in one technological cycle of chip manufacturing will allow creating "system-on-chip", providing both power and low-signal parts.

The task of ensuring the integration of e-mode PHEMT to the manufactured d-mode PHEMT on the available technological equipment for the possibility of making a new class of devices on gallium nitride is defined at the Institute of Ultra-High-Frequency Semiconductor Electronics, RAS. According to the literature, the most common methods of obtaining normally-off transistors on the AlGaN/GaN are fluorine (F-) implantation into the gate region[1]-[3], using thin structures[4], formation of the p-type GaN region under the gate[6]-[8], etching of the AlGaN barrier layer under the gate[9]-[15].

\section{METHODS OF OBTAINING OF NORMALLY-OFF TRANSISTORS ON ALGAN/GAN}

\section{A. Implantation of fluorine into the gate region}

The formation of normally-off transistors using a fluorine-implanted region under the gate realized by plasma treatment of the surface of the barrier layer in fluorine-containing plasma before the gate metallization.

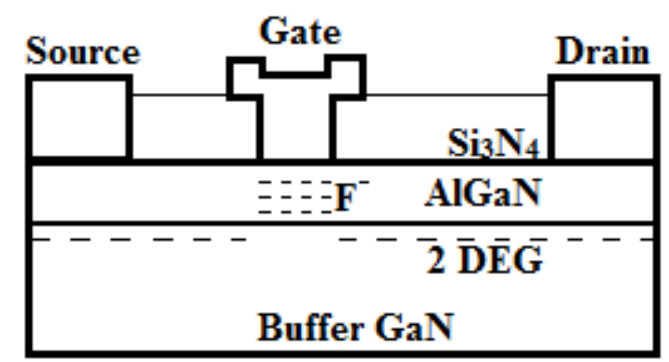

Fig. 1. Schematic representation of a normally-off transistor to the implanted region under the gate metallization

The most common fluorine-containing gas carbon tetrafluoride (CF4) is used in this method. Plasma with energies sufficient to make the fluorine penetrate into the surface region of the heterostructure is used. The efficiency of using implantation of fluorine in $\mathrm{GaN}$ is due 
to its strong electronegativity. In this case, single fluorine ions in the near-surface layer tend to capture free electrons and become strongly negatively charged. These charges modulate the local potential which impoverishes the channel with a 2 DEG. Fig.1 schematically shows the production of a normally-off transistor with fluorine implantation in the gate metallization.

The technology of fluorine implantation into the gate region allows to realize analog, power and digital parts on single crystal, by manufacturing of D-mode HEMT and Emode HEMT [2]. However, partial etching of the barrier layer of the heterostructure and the introduction of additional radiation defects during the fluorine implantation affects the electrophysical characteristics. It is noted that the use of plasma in $\mathrm{CF}_{4}$ with different energy allows obtaining a recessed gate in the barrier layer with fluorine implanted under the gate metallization [3].

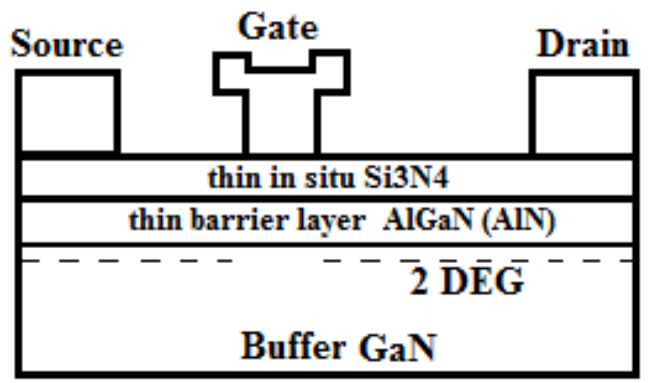

Fig. 2. Schematic representation of a normally-off transistor with a thin barrier layer

\section{B. The use of thin heterostructures}

The use of thin heterostructures allows forming a normally-off transistor due to the proximity of the transistor channel to the gate. The technology of molecular beam epitaxy of SiC/AlN/GaN heterostructures with ultrathin barrier passivated in the growth chamber was developed in Novosibirsk. The thickness of the ultrathin barrier was about 4-5 nm, which allowed to obtain a transistor operating in the enrichment mode [5]. Fig. 2 schematically shows normally-off transistor on a thin heterostructure. Thus, the making of a normally-off transistor is determined by the making of a specific structure with a thin barrier layer. Growth of such structures requires compromise conditions for the formation of the upper part of the AlN/GaN heterostructure. On such structures, the implementation of power and low-signal parts of the chip will not work while it`s impossible to make normally open transistors on these structures..

\section{Using p-GaN in gate region}

The use of structures doped with magnesium GaN (p$\mathrm{GaN} / \mathrm{AlGaN} / \mathrm{GaN}$ ) implies the selective removal of the $\mathrm{p}$ $\mathrm{GaN}$ layer before the formation of ohmic contacts. Fig.3 schematically shows normally-off transistor with a p-GaN area under the gate. The gate threshold voltage of a transistor with a sub-gate region based on $\mathrm{p}-\mathrm{GaN}$ is determined, mainly, by the thickness of the epitaxial layer of $\mathrm{p}-\mathrm{GaN}$ and its doping level. Optimization of these parameters allows to achieve a threshold gate voltage of $\mathrm{GaN}$-transistors close to $\mathrm{Vth}=+2 \mathrm{~V}$. Due to the use of the MIS structure in the $\mathrm{p}-\mathrm{GaN}$ transistor, the gate threshold voltage increases to $\mathrm{Vth}=+6.8 \mathrm{~V}[8]$.

\section{Depth into the barrier layer (recession)}

The most common and easy-to-implement method for obtaining a normally-off transistor on a AlGaN/GaN is the use of a gate deepening (recession) into the barrier layer of AlGaN [9], [10]. This technology is most common in arsenide-gallium technology, due to the realization of the recession by liquid chemical etching in solutions. The use of stop layers allows to carry out the unchecked etching process protective layers of $\mathrm{GaAs} / \mathrm{AlGaAs}$ and provides the necessary homogeneity. In AlGaN / GaN is necessary to deepen into AlGaN without using stop layers. Process of chemical etching in solution is not implemented due to the strong chemical bond between nitrogen and gallium. Therefore, so-called "dry" etching methods are used, with low energies of charged particles in a chlorine-containing environment. Usually, for the implementation of a normally-off transistor with a recession, at first formed an instrument isolation, ohmic contacts to the heterostructure, then a plasma chemical etching of the gate recession is carried out through the mask. Transistor manufacturing ends with lithography and $\mathrm{Ni} / \mathrm{Au}$ gate metallization. Normally-off transistor with gate deepening schematically shown in fig.4.For high-power transistors after recess, a thin gate dielectric is deposited by the deposition of $\mathrm{Al}_{2} \mathrm{O}_{3}$ or $\mathrm{Si}_{3} \mathrm{~N}_{4}$ films by means of chemical vapor deposition or ALD. The advantages of such transistors include a high threshold gate voltage close to $\mathrm{Vth}=+5 \mathrm{~V}$.

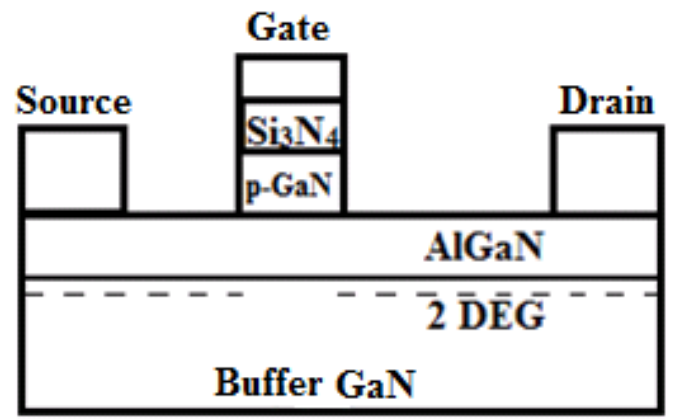

Fig. 3. Schematic representation of a normally-off transistor with a $\mathrm{p}-\mathrm{GaN}$ region under the gate

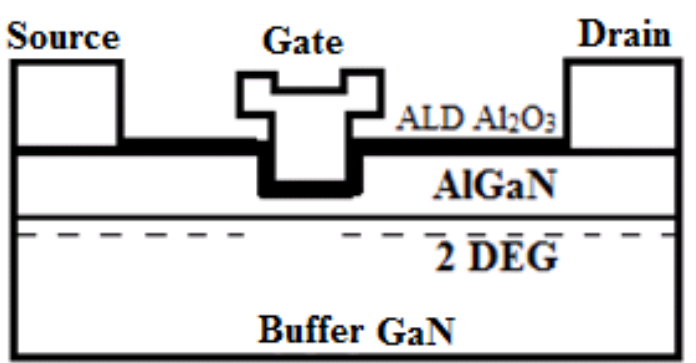

Fig. 4. Schematic representation of a normally-off transistor using a gate recess

Also, there are works in which the methods of obtaining normally-off transistors on GaN are combined. 
The formation of a gate groove is carried out in a chlorinecontaining environment, followed by treatment in fluorine-containing plasma, forming an area implanted with fluorine under the gate. This will reduce gate-drain leakage currents and improve the reliability and efficiency of such transistors [11].

There is a plasma-chemical effect on the barrier layer of ions of different energies in the manufacture of normally-off transistors with a gate penetration on $\mathrm{GaN}$, which leads to the formation of radiation defects. Reducing the thickness of the barrier layer to a few nanometers leads to the appearance of defects in the nearsurface region, which affect the electrophysical properties of the heterostructure, which ultimately affects the parameters of the microwave transistors and their reliability. Methods of digital etching of the barrier layer of AlGaN are developed to reduce these effects in the formation of the gate.

\section{DiGITAL ETCHING OF THE BARRIER LAYER OF AlGaN}

Digital etching [12] is a two-stage etching process: semiconductor oxidation and removal of its oxide. In one etching cycle, a certain thickness of the semiconductor is removed and several cycles are performed to achieve a controlled thickness. An oxygen environment is used to oxidize the AlGaN / GaN heterostructure, and a chlorinecontaining environment is used to remove the oxidized AlGaN barrier layer. This sequential removal of oxidized layers provides a sinking rate of 1.5-2.5 nm per cycle. The idea of the two-stage plasma-chemical etching method for $\mathrm{GaN}$ transistors operating in the E-mode is patented by the Hrl laboratory and described in detail in [12]. In this work shown the comparison of normally-off transistors, made with the gate depth "digital" etching, with transistors, fabricated with the implantation of fluorine in the gate region. For the same heterostructures designs transistors that were fabricated using digital etching (Fig. 4) had a gain slope (GM) of about $250 \mathrm{MS} / \mathrm{mm}$, which is $67 \%$ more than for transistors that were fabricated with fluorine implantation (Fig. 1).The threshold gate voltage variation for transistors with digital etching two times more than for transistors with fluorine implantation. Breakdown voltage for transistors with such recession was more than $1000 \mathrm{~V}$.

To carry out the process of digital etching AlGaN a hard mask is formed from dielectric film, through which the oxidation and subsequent removal of the oxidized layer AlGaN can be achieved. After completing the required number of cycles, the dielectric film is removed, a thin film of the passivating dielectric is deposited and a transistor gate is formed. A system with a source of highfrequency (HF) power, implementing the method of reactive ion etching, was used for oxidation and etching of oxides. During the processes, plasma with low HF power was used, which was effective in removing oxides. Replacing reactive chlorine with boron trichloride improved the selectivity in removing the oxide. The reproducibility of the process is confirmed by the linear dependence of the height of the formed step on the number of digital etching cycles. But use of reactive ion etching modes, high-energy ions are present in the plasma, which lead to the formation of radiation defects and partial etching because of the physical component of the AlGaN barrier itself when the oxide is removed.

Due to the appearance of radiation defects during etching of barrier layers and its oxides in recent works on "digital" etching AlGaN, began to refuse the "dry" etching and oxides are removed by liquid chemical etching in hydrochloric acid solutions [13], [14]. Due to the low etching rate (about $0.5 \mathrm{~nm}$ per cycle) liquid etching for removing oxides in the "digital" etching can only be used for thin barrier layers. The process in this case is too consuming because of the need to use multiple plants for one etching cycle.

To implement the "digital" etching by the "dry" method , a defect-free low-energy etching of the AlGaN barrier layer was developed in IHFUSE RAS. This method allows removing the oxidized layer without generating radiation defects [15]. The proposed technology allows implementing d-mode PHEMT and emode PHEMT in one technological cycle of chip making. The output characteristics of the manufactured nitride transistors on a sapphire substrate are shown on fig.5.

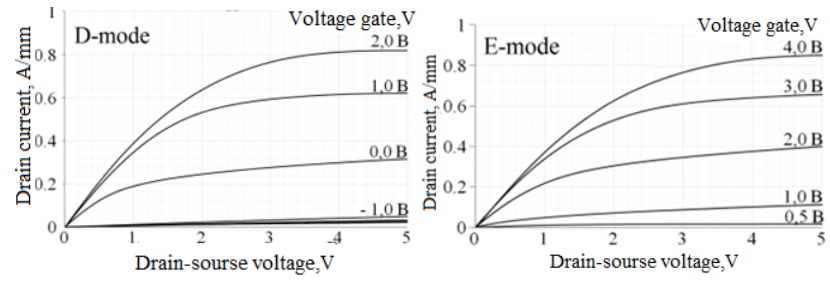

Fig. 5. The output characteristics of the transistors operating in the (E-mode) and (D-mode)

\section{LOW-ENERGY DEFECTLESS DRY ETCHING AIGaN BARRIER}

According to the proposed digital etching technology, the oxidation of AlGaN barrier layer in oxygen containing plasma and the low-energy defectless etching of the oxide are carried out in the same chamber without extracting and recharging substrates. The entire process was performed in a Sentech SI-500 inductively-coupled plasma (ICP) etching system. Use of the ICP source ensures the generation of high density plasma with low energies of charged particles interacting with the processed surface, which prevents the formation of radiation-induced defects in the near-surface layers of transistor heterostructures. In addition, a high concentration of charged particles in the reactor during plasma chemical processes ensures high velocity and quality of etching. During both the oxidation of barrier and the etching of oxide, the ICP source was used together with a high-frequency (HF) electrode, which ensured the reactive ion etching in an inductively-coupled plasma (RIE-ICP) regime that allowed controlled variation of the bias voltage on the HF electrode. A previous investigation [11] showed that defect formation during the RIE-ICP processing of dielectrics and semiconductors is minimized if the bias voltage on the HF electrode does not exceed $40 \mathrm{~V}$. However, it was recently 
reported that oxide-layer etching on the surface of a nitride-based heterostructure in RIE-ICP regime at an HF electrode voltage below $20 \mathrm{~V}$ prior to the formation of ohmic contacts probably did not ensure the removal of a parasitic $\mathrm{B}_{\mathrm{x}} \mathrm{Cl}_{\mathrm{y}}$ film formed during the plasma chemical treatment of $\mathrm{GaN}$ by ions of $\mathrm{BCl}_{3}$ plasma and this, in turn, hindered complete removal of an oxide layer from the heterostructure surface. With allowance for this circumstance, we have selected a RIE-ICP regime for etching the oxide film formed during oxygen-containing plasma interaction with the AlGaN barrier layer surface, such that the HF electrode bias voltage was within 20$40 \mathrm{~V}$. The ICP source power amounted to $100 \mathrm{~W}$ and the $\mathrm{HF}$ generator power was $10 \mathrm{~W}$ at a $\mathrm{BCl}_{3}$ pressure in the reaction chamber on the order of $1 \mathrm{~Pa}$ and a gas-flow rate of $10 \mathrm{sccm}$. Under these conditions, the HF electrode bias voltage was about $25 \mathrm{~V}$, which ensured defectless selective etching of the oxide film. The selectivity was confirmed by the absence of AlGaN etching traces after long-term processing in this RIE-ICP regime via a dielectric mask. One digital etching cycle removed the approximately 1.5-nm thick AlGaN layer.

In addition, the proposed technology makes it possible to create $\mathrm{AlGaN} / \mathrm{AlN} / \mathrm{GaN} / \mathrm{Al}_{2} \mathrm{O}_{3}$ (D- and E-type) on the one wafer. Plots of the transconductance and drain current vs. gate voltage on the Fig.6. Cutoff voltage for D-mode HEMT $-1.3 \mathrm{~V}$, barrier voltage for E-mode $0.5 \mathrm{~V}$.

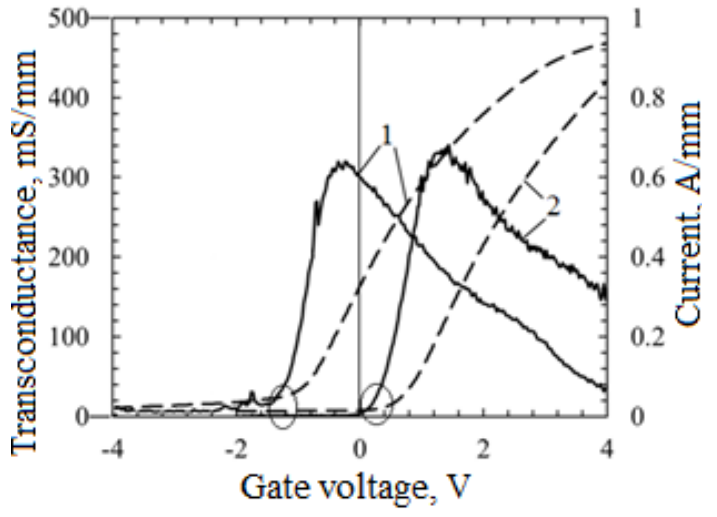

Fig. 6. Plots of the transconductance (solid curves) and drain current (dashed curves) dependence on gate voltage for $D$ mode (1) and E-mode(2)

\section{CONCLUSION}

The most appropriate method of realization normallyoff transistors in a single technological cycle is the formation of gate deepening. In IHFUSE RAS for the formation of the gate deepening was developed "digital" etching by the "dry" method, of the AlGaN barrier layer. This method allows removing the oxidized layer without generating radiation defects. This method can be easily integrated into the technological cycle of manufacturing chips on GaN. Realization of d-mode PHEMT and e-mode PHEMT in one technological cycle will allow to creating "system-on-chip", providing both power and low-signal parts.

\section{SUPPORT}

The reported study was funded by RFBR according to the research project № 18-07-01426 A.

\section{REFERENCES}

[1] TangZ., JiangQ., Lu Y., HuangS., YangS., TangX., and ChenK. J. $600-\mathrm{V}$ normally off $\mathrm{SiN}_{\mathrm{X}} / \mathrm{AlGaN} / \mathrm{GaN}$ MISHEMT with large gate swing and low current collapse // IEEE Electron DeviceLett. 2013. V. 34. № 11, P. 13731375.

[2] Chen K.J., Kwan A.M.H., Jiang Q. Technology for III-N heterogeneous mixed-signal electronics // Physica Status Solidi A.2014. V.211№ 4 P. 769-774.

[3] Liu C., Yang S., Liu S., Tang Z., Wang H., Jiang Q., Chen K.J. Thermally stable enhancementmodeGaN metalinsulator-semiconductor high-electron-mobility transistor with partiallyrecessed fluorine-implanted barrier // IEEE Electron Device Lett. 2015. V. 36. № 4. P. 318-320.

[4] Brown R., Macfarlane D., Al-Khalidi A., Li X., Ternent G., Zhou H., Thayne I. and Wasige E.A sub-critical barrier thickness normally-offAlGaN/GaN MOS-HEMT // IEEE Electron Device Lett. 2014. V. 35. № 9. P. 906-908.

[5] Zhuravlev K.S., Malin T.V., Mansurov V.G., Tereshenko O.E., Abgaryan K.K., Reviznikov D.L., Zemlyakov V.E., Egorkin V.I., Parnes Y.M., Tikhomirov V.G., Prosvirin I.P. AlN/GaN heterostructures for normally-offtransistors // Semiconductors. 2017. V. 51. №. 3. P. 395-402. (in Russian)

[6] Meneghini M., Hilt O., Wuerfl J. and Meneghesso G. Technology and reliability of normally-off GaNHEMTs with p-type Gate // Energies. 2017.V. 10. № 2. 153

[7] Wang H., Wei J., Xie R., Liu C., Tang G. and Chen K. J. Maximizing the performance of $650-\mathrm{V}$ p-GaN gate HEMTs: dynamic Rondegradation and circuit design considerations // IEEE Transactions on Power Electronics. 2017. V. 32. № 7. P. 5539-5549.

[8] Erofeev E.V., Fedin I.V., Fedina V.V., Stepanenko M.V., Yuryeva A.V. High-voltage MIS-gate transistors // Semiconductors. 2017. V 51. № 9. P.1278-1281. (in Russian)

[9] Lanford W. B., Tanaka T., Otoki Y. and Adesida I.Recessed-gate enhancement-mode GaN HEMT with high threshold voltage // Electronics Lett. 2005. V. 41. № 7. P. 449-450.

[10] Recessed-gate structure approach toward normally off highvoltage $\mathrm{AlGaN} / \mathrm{GaN}$ HEMT for power electronics applications // IEEE Transactions on Electron Devices. 2006. V. 53. № 2. P.356-362.

[11] Lin J.-H., Huang S.-J., Lai C.-H. and Su Y.-K. Normallyoff $\mathrm{AlGaN} / \mathrm{GaN}$ high-electron-mobility transistor on $\mathrm{Si}(111)$ by recessed gate and fluorine plasma treatment // Japanese Journal of Applied Physics. 2016. V. 55. № 1S. P. 01AD05.

[12] Burnham S. D., Boutros K., Hashimoto P., Butler C., Wong D.W.S., Hu M. and Micovic M. Gate-recessed normally-off GaN-on-Si HEMT using a new $\mathrm{O}_{2}-\mathrm{BCl}_{3}$ digital etching technique// Physica Status Solidi C. 2010. V. 7. № 7-8. P.2010-2012.

[13] Yamanaka R., Kanazawa T., Yagyu E. and MiyamotoY. Normally-off AlGaN/GaN high-electron-mobility transistor using digital etching technique // Japanese Journal of Applied Physics. 2015. V. 54. № 6. P. 06FG04. 
[14] Chakroun A., Jaouad A., Bouchilaoun M., Arenas O., Soltani A. and MaherH.Normally-off AlGaN/GaN MOSHEMTusing ultra-thin $\mathrm{Al}_{0.45} \mathrm{Ga} 0.55 \mathrm{~N}$ barrierlayer // Physica Status Solidi A. 2017. V. 214. № 11. P. 1600836.

[15] Mikhailovich S.V., Pavlov A.Y., Tomosh K.N., Fedorov Y.V. Low-energy defect-free dry etching of the barrier layer AlGaN/AlN/GaNHEMT // Technical Physics Lett. 2018. V. 44. №. 10. P. 61-67. (in Russian)

[16] Tomosh K.N., Pavlov A.Y., Pavlov V.Y., Khabibullin R.A., Arutyunyan S.S., Maltsev P.P. Investigation of the fabrication processes of AlGaN/AlN/GaN HEMTs with in situ $\mathrm{Si}_{3} \mathrm{~N}_{4}$ passivation // Semiconductors. 2016. V. 50. №. 10. P. 1434-1438. (in Russian)

[17] Andrianov N.A., Kobelev A.A., Smirnov A.S., Barsukov Y.V., Zhukov Y.M. Influence of surface processing in a $\mathrm{BCl} 3$ plasma on the formation of ohmic contacts to AlGaN/GaN structures // Technical Physics. The Russian Journal of Appled Physics. 2017. V. 87. №. 3. P. 413-418. (in Russian) 